\title{
On the existence of a generalized solution to the first initial-boundary value problem for a nonlinear parabolic equation
}

\author{
V. SEDA (Bratislava, Czechoslovakia) (*)
}

Summary. - In the paper first the existence of a classical solution to an initial-boundary value problem for the nonlinear parabolic equation $\frac{\partial z}{\partial t}=\frac{\partial^{2} z}{\partial x^{2}}+f(x, t, z)$ is proved under the standart condition on Hölder continuity of $f$ but a quite general condition on the growth of $f$. Then, by using the possibility of the approximation of a continuous fun. ction by means of Holder continuous functions, the foregoing result is applied to the proof of the existence of a generalized solution to the first boundary value problem for the same equation where only continuity of $f$ and a weak assumption on the growth of $f$ is required.

1. - The existence of a solution to an initial-boundary value problem for the nonlinear parabolic equation $\frac{\partial z}{\partial t}=\frac{\partial^{2} z}{\partial x^{2}}+f(x, t, z)$ (also in more general form) was already proved by many authors and in different ways. The result as well as the method to obtain it mainly depend on the class of functions to which the expected solution has to belong. When a classical solution is searched, then $f$ is subjected to a Hölder condition and to a restriction concerning its growth. (See e.g. [1], [2], [3], [9]). The existence of a generalized solution requires only mild conditions on $f$ concerning its continuity and measurability. Still a restriction on the growth of $f$ remains. (See [5], [6]). The review of the applied methods and the obtained results can be found e.g. in $[3],[4],[7],[10])$.

The aim of the present paper is to assure the existence of a generalized solution to the mentioned problem assuming the continuity of $f$ and a fairly general condition on the growth of $f$. The idea of the proof consists of approximating $f$ by a sequence of Hölder continuous functions and thus, of approximating the given initial-boundary value problem by a sequence of similar problems for which the elassical solution $z_{n}$ can be found. Then the uniform limit of $z_{n}$ exists and gives the sealched generalized solution. The structure of that solution is also given.

(*) Entrata in Redazione il 14 giugno 1970 
We shall start with some properties of Hölder continuous functions. Let $(K, \rho)$ be a metric space, $\emptyset \neq M \subset R$ be a set, and let $d(M)$ be the diameter of $M$. Let us denote by $H_{\alpha}(M)$ the set of allüblder continuous functions on $M$ of exponent $\alpha, 0<\alpha \leqq 1$. Thus $f \in H_{\alpha}(M)$ iff $f$ is defined on $M$ and there exists a constant $L=L(f)>0$ such that for any two points the inequality

$$
|f(y)-f(x)| \leqq L[p(x, y)]^{\alpha}
$$

is true. Here as well as in what follows only real functions are considered. Further the following reinark and theorems will be of use.

Remark. - If $d(M)<+\infty$, and $\beta$ satisfies the inequalities $0<\alpha \leqq \beta \leqq 1$, then from the relation $[\rho(x, y)]^{2} \leqq[\rho(x, y)]^{\alpha}$ and $[\rho(x, y)]^{\beta} \leqq d^{\beta-x}[\rho(x, y)]^{\alpha}$, respectively, which is valid for $\rho(x, y) \leqq 1$ and $1<\rho(x, y) \leqq d$ (if $d>1$ ), respectively, it follows that

$$
H_{\beta}(M) \subset H_{\alpha}(M)
$$

Theorem 1. - Let $M$ be a compact set, $\alpha$ be a real number satisfying the incqualities $0<\alpha \leqq 1$ and $f \in C_{0}(M)$. Let further the functions $F_{1}, F_{2} \in$ $H_{\alpha}(M)$ and be such that $F_{1}(x) \leqq f(x) \leqq F_{2}(x)$ for each $x \in M$. Then there exists a sequence $f_{n} \in H_{\alpha}(M), n=1,2,3, \ldots$, which is uniformly convergent to $f$ on $M$ whereby

$$
F_{1}(x) \leqq f_{n}(x) \leqq F_{2}(x)
$$

for each $x \in M$ and all natural $n$.

Theorem 2. - Let $R$ be a locally compact separable metric space and $\left\{M_{m}\right\}_{m=1}^{\infty}$ an arbitrary increasing sequence of compact sets in $R$ such that $R=\bigcup_{m=1}^{\infty} M_{m}$. Let the number $\alpha$ satisfy the inequalities $0<\alpha \leqq t$. Let the function $f \in C_{0}(R)$. Then there exists a sequence $\left\{f_{n}\right\}_{n=1}^{\infty}$ of the functions $f_{n} \in H_{x}(R)$ which is uniformly convergent to $f$ on each $M_{m}, m=1,2,3, \ldots$.

If moreover there exist two functions $F_{1}, F_{2}$ such that $F_{1}, F_{2} \in H_{\alpha}(R)$ (for each $m=1,2,3, \ldots F_{1}, F_{2} \in H_{\alpha}\left(M_{m}\right)$ ) and $F_{1}(x) \leqq f(x) \leqq F_{2}(x)$ for every $x \in R$, then the sequence $\left\{f_{n}\right\}_{n=1}^{\infty}$ satisfies the inequalities (3) for each $x \in R$ and all natural $n$ the sequence $\left\{f_{n}\right\}_{n=1}^{\infty}$ satisfies the inequalities (3) for each $x \in R$ and all natural $n$, but instead of $f_{n} \in H_{\alpha}(R)$ it is only true that $f_{n} \in H_{x}\left(M_{m}\right)$ for each $\boldsymbol{m}=1,2,3, \ldots$ ).

Note. - The proof of Theorems 1 and 2 will be given in another paper. By approximating a continuous function by means of polsnomials we obtain Theorems 1 and 2 only in a special case when $F_{1} \leqq f-\varepsilon, F_{2} \geqq f+\varepsilon, \varepsilon>0$. 
Although this case is sufficient for the proof of Theorem 3, in Lemma 5 a continuous $f$ satisfying the inequality (10) is approximated by means of $f_{n} \in H_{\alpha}$ satisfying (19). Hence Theorem 2 is applied in a more general case which does not follow from the Weierstrass theorem.

2. - We are now going to apply the above mentioned results. As a locally compact separable metric space we shall take the $n$-dimensional euclidean space $E_{n}$. Further for given $a>0, b>0$ and $0 \leqq k \leqq l$ integers we define the sets $B_{k, l}, B$ in $E_{2}$ by the relation $B_{k, l}=\{(t, y): 0 \leqq t \leqq b, k \leqq y \leqq l\}, B=\{(t, y)$ : $: 0 \leqq t \leqq b, 0 \leqq y<+\infty)$ and the sets $D_{k}, D$ in $E_{3}$ by $D_{k}=\{(x, t, y): 0 \leqq x \leqq a$, $0 \leqq t \leqq b,-k \leqq y \leqq k\}, D=\{(x, t, y): 0 \leqq x \leqq a, 0 \leqq t \leqq b,-\infty<y<+\infty\}$. Finally, let $E=\{(x, t): 0 \leqq x \leqq a, 0 \leqq t \leqq b)$ and let $E_{0}$ denote the interior of $E$. In the last part of the paper we shall consider the space $W^{2,1}(E)$ which means the set of all functions $w$ which together with their weak derivatives $\frac{\partial u}{\partial x}, \frac{\partial^{2} u}{\partial x^{2}}, \frac{\partial u}{\partial t}$ belong to $L^{2}(E)$. The norm in this linear space is given by $|u|_{2,1}=\left\{\iint_{E}\left[u^{2}(x, t)+\left(\frac{\partial u(x, t)}{\partial t}\right)^{2}+\left(\frac{\partial u(x, t)}{\partial x}\right)^{2}+\left(\frac{\partial^{2} u(x, t)}{\partial x^{2}}\right)^{2}\right] d x d t\right\}^{\frac{1}{2}}$ (compare with [4], p. 15). Lemma 6.2 ([4], p. 16) remains to be true in this space.

Similarly as in [9], p. 257, we shall say that the function $p=p(t, y)$ sa. tisfies the condition $(A)$ if $p \in C_{0}(B)$ and for any $c \geqq 0$ the right maximal integral $y(t, c)$ of the differential equation

$$
y^{\prime}=p(t, y)
$$

such that

$$
y(0, c)=c
$$

exists on the whole interval $\langle 0, b\rangle$.

From the property of the differential equation (4) that their solutions form a lattice it follows that if $0 \leqq c_{1}<c_{2}$, then $y\left(t, c_{1}\right) \leqq y\left(t, c_{2}\right)$ on the intersection of their intervals of definition. Hence for $n-1<c<n y(t, n-1) \leqq y(t, c) \leqq$ $y(t, n)$ on the common part of their intervals of definition and the condition (A) will be satisfied if for each $n=0,1,2, \ldots y(t, n)$ exists on the whole in. terval $\langle 0, b\rangle$. If moreover $p(t, y) \geqq 0$ for all $(t, y) \in B$, it suffices to prove the existence of $y(t, x)$ on the interval $\langle 0, b\rangle$ only for all natural $n$.

Theorem 3. - Let $p$ satisfy the condition $(A)$ and $p(t, y) \geqq 0$ in $B$. Then there exists a function $q$ which also satisfies the condition $(A)$ whereby $q \in H_{1}\left(B_{0, m}\right)$ for each $m=1,2,3, \ldots$ and $q(t, y)>p(t, y)$ in $B$. 
Proof. - For each natural $n$, on the basis of Theorem 2, [8], p. 83, there exists an $\varepsilon_{n}>0$ such that the right maximal solution of the problem $y^{\prime}=$ $p(t, y)+\varepsilon_{n}, y(0)=n$, exists in $\langle 0, b\rangle$. By using the Comparison theorem 1, [8], p. 82, we may assume that $\varepsilon_{n} \geqq \varepsilon_{n+1}$ for all $n$. Further from Theorem 1 the existence of a function $q_{n} \in H_{1}\left(B_{n-1, n}\right)$ in $B_{n-1, n}$ follows which satisfies the inequalities

$$
p(t, y)+\frac{\varepsilon_{n}}{4}<q_{n}(t, y)<p(t, y)+\frac{3}{4} \varepsilon_{n},(t, y) \in B_{n-1, n}
$$

By the uniform continuity of $p$ in $B_{n-1, n}$ there exists such $y_{n}, n-1<y_{n}$ $<n$ that

$$
p(t, n)-\frac{\varepsilon_{n+1}}{8}<p(t, y)<p(t, n)+\frac{\varepsilon_{n+1}}{8}, 0 \leqq t \leqq b, y_{n} \leqq y \leqq n
$$

Let $q$ be the function defined in $B$ in the following way:

$$
q(t, y)=\left\{\begin{array}{l}
q_{n}(t, y), 0 \leqq t \leqq b, n-1 \leqq y \leqq y_{n} \\
q_{n}\left(t, y_{n}\right)+Q_{n}(t)\left(y-y_{n}\right), 0 \leqq t \leqq b, y_{n} \leqq y \leqq n,
\end{array}\right.
$$

where $Q_{n}(t)=\left[q_{n+1}(t, n)-q_{n}\left(t, y_{n}\right)\right] /\left(n-y_{n}\right)$. By this definition $q$ satisfies the inequalities (6) for $0 \leqq t \leqq b, n-1 \leqq y \leqq y_{n}$ (with $q_{n}(t, y)=q(t, y)$ ). As to the behaviour of $q$ on the set $0 \leqq t \leqq b, y_{n} \leqq y \leqq n$, two cases may happen.

When $q_{n}\left(t, y_{n}\right) \leqq q_{n+1}(t, n)$, in virtue of $(7)$ and $(6)$ we have $p(t, y)<p(t, n)+$ $\frac{\varepsilon_{n+1}}{8}<p\left(t, y_{n}\right)+\frac{\varepsilon_{n+1}}{4}<q_{n}\left(t, y_{n}\right) \leqq q(t, y) \leqq q_{n+1}(t, n)<p(t, n)+\frac{3}{4} \varepsilon_{n+1}<p(t, y)+\frac{7}{8} \varepsilon_{n+1}$.

When $q_{n}\left(t, y_{n}\right)>q_{n+1}(t, n)$, it is true that $p(t, y)+\frac{\varepsilon_{n+1}}{8}<p(t, n)+\frac{\varepsilon_{n+1}}{4}<$ $q_{n+1}(t, n) \leqq q(t, y) \leqq q_{n}\left(t, y_{n}\right)<p\left(t, y_{n}\right)+\frac{3}{4} \varepsilon_{n}<p(t, n)+\frac{3}{4} \varepsilon_{n}+\frac{1}{8} \varepsilon_{n+1}<p(t, y)+\varepsilon_{n}$.

Altogether we have got that in $B_{n-1, n}$ for $q$ the following inequalities are true

$$
p(t, y)<q(t, y)<p(t, y)+\varepsilon_{n} .
$$

Further for $0 \leqq t \leqq b, y_{n} \leqq \bar{y}<\bar{y} \leqq n,|q(t, \bar{y})-q(t, \bar{y})| M|\overline{\bar{y}}-\bar{y}|$ because $Q_{n}(t)$ is continuous in $\langle 0, b\rangle$. On the other hand for $0 \leqq t_{1}<t_{2} \leqq b, y_{n} \leqq y$ $\leqq n,\left|q\left(t_{2}, y\right)-q\left(t_{1}, y\right)\right| \leqq\left(L_{n}+\left(y-y_{n}\right)\left(L_{n+1}+L_{n}\right) /\left(n-y_{n}\right)|| t_{2}-t_{1} \mid\right.$, where $L_{n}$ is the Lipschitz constant for the function $q_{n}$ in $B_{n-1, n}$. This, together with the properties of $q_{n}$, gives that $q$ satisfies a Lipschitz condition in $B_{n-1, n}$ and hence also in $B_{0, n}$. With respect to the inequalities (8) on the basis of the Comparison theorem $1,[8]$, p. 82 , there exists the right maximal solution of 
the problem $y^{\prime}=g(t, y), y(0)=n$ for each natural $n$ and thus, using the remark before Theorem 3 , the function $q$ satisfies the condition $(A)$.

3. - The next step in proving the existence of a generalized solution will be the extension in a certain sense of the validity of the existence the orem proved in [9]. (Compare also with the existence theorem in [2], p. 314). To that aim (similarly as in [9]. p. 259) we shall say that the function $f=$ $f(x, t, y)$ defined in $D$ satisfies the condition $(H)$ if for each point $(x, y), 0<$ $x<a,-\infty<y<+\infty$ there exist a neighborhood $N(x, y)$ of that point and constants $L>0,0<\alpha<1,0<\beta<1$ depending in general on $(x, y)$ such that for $\overline{(x,}, \bar{y}),(\overline{\bar{x}}, \overline{\bar{y}}) \in N(x, y)$ and arbitrary $0 \leqq t \leqq b$ the inequality $\mid f(\bar{x}, t, \bar{y})$ $f(\overline{\bar{x}}, t, \overline{\bar{y}}) \mid \leqq L\left(|\bar{X}-\overline{\bar{X}} \alpha+| \bar{y}-\overline{\bar{y}}\left({ }^{\beta}\right)\right.$ holds .

Further the solution of the first initial-boundary value problem

$$
\begin{gathered}
\frac{\partial u}{\partial t}=\frac{\partial^{2} u}{\partial x^{2}} \quad 0<x<a, 0<t<b \\
u(x, 0)=\chi(x), \quad 0 \leqq x \leqq a, \\
u(0, t)=\varphi(t), \quad u(a, t)=\psi(t), 0 \leqq t \leqq b
\end{gathered}
$$

where $\chi \in C_{0}(<0, a>), \varphi, \psi \in C_{0}(<0, b>)$, and $\chi(0)=\varphi(0), \chi(a)=\psi(0)$, will be denoted by $u=u(x, t ; \varphi, \chi, \psi)$ (compare with [11]). The following tbeorem is true.

Theorem 4. - Suppose that the function $p=p(t, y)$ satisfies the condition $(A)$, the function $f=f(x, t, y)$ is continuous in $D$, satisfies the condition $(H)$ and

$$
|f(x, t, y)| \leqq p(t,|y|),(x, t, y) \in D
$$

Let further the functions $\varphi$ : $\psi \in H_{a}(<0, b>), \chi \in H_{\alpha}(<0, a>)$ for an $\alpha$, $0<\alpha<1$ and $\chi(0)=\varphi(0), \chi(a)=\psi(0)$. Then there exists at least one solution $z$ of the differential equation

$$
\frac{\partial z}{\partial t}=\frac{\partial^{2} z}{\partial x^{2}}+f(x, t, z) \quad 0<x<a, 0<t<b,
$$

satisfying the conditions

$$
\begin{gathered}
z(0, t)=\nsucc(t), z(a, t)=\psi(t), 0 \leqq t \leqq b, \\
z(x, 0)=\chi(x), 0 \leqq x \leqq a .
\end{gathered}
$$

Moreover there exists a constant $M>0$ such that for any two points 
$\left(x_{1}, t_{1}\right),\left(x_{2}, t_{2}\right) \in E$ the solution $z$ satisfies the inequality

$$
\left.\left|z\left(x_{2}, t_{2}\right)-z\right| x_{1}, t_{1}\right) \mid \leqq M\left(\left|x_{2}-x_{1}^{\prime \alpha}+\right| t_{2}-\left.t_{1}\right|^{\alpha / 2}\right) .
$$

The proof of this theorem will be based on Lemmas either stated or proved in the papers [9], [11] and [2].

Lemma 1 ([9], p. 257). - Assume that the function $p=p(t, y)$ satisfies the condition $(A)$, the function $f=f(x, t, y)$ is defined in $D$ and satisfies the inequality $(10)$ in $D$. Suppose that $v=v(x, t)$ is continuous in $E$ and possesses the continuons derivative $\frac{\partial^{2} v}{\partial x^{2}}$ in the interior of $E$. Assume that $v$ satisfies in interior of $E$ the equation (11) and the boundary inequalities

$$
\begin{gathered}
|v| 0, t)|\leqq c, \quad| v(a, t) \mid \leqq c, \quad 0 \leqq t \leqq b \\
|v(x, 0)| \leqq c, \quad 0 \leqq x \leqq a
\end{gathered}
$$

for some $c \geqq 0$. Under these assumptions we have the inequality

$$
|v(x, t)| \leqq y(t, c),(x, t) \in E
$$

where $y(t, c)$ is the right maximal integral of the differential equation (4) satisfying (5).

Lemma 2 ([2], p. 301). - For any $\gamma, 0<\gamma<1$, there exists a constant $c(\gamma)>0$ such that for any funetion $g=g(x, b) \in C_{0}(E)$ satisfying the inequality $|g(x, t)| \leqq M$ for $(x, t) \in E$ it is true: If

$$
\begin{gathered}
p(x, t)=\frac{1}{2 \sqrt{\pi}} \int_{0}^{t} \int_{0}^{a} \frac{\exp \left[-(x-\xi)^{2} / 4(t-\tau)\right]}{\sqrt{t-\tau}} g(\xi, \tau) d \xi d \tau, \quad 0 \leqq x \leqq a, 0<t \leqq b, \\
p(x, 0)=0,0 \leqq x \leqq a,
\end{gathered}
$$

then for any two points $\left(x_{1}, t_{1}\right),\left(x_{2}, t_{2}\right) \in E$ we have

$$
\left|p\left(x_{2}, t_{2}\right)-p\left(x_{1}, t_{1}\right)\right| \leqq c(\gamma) M\left(\left|x_{2}-x_{1}\right|+\left|t_{2}-t_{1}\right| \frac{1+\gamma}{2}\right) .
$$

Lemma 3 ([2], p. 302). - For any $\sigma, 0<\sigma<\frac{1}{2}$, there exists a constant $r(\sigma)>0$ such that if the functions $\varphi$ and $\psi \in C_{0}(\langle 0, b\rangle), \varphi(0)=\psi(0)=0$, satisfy a Hölder condition $\left|\varphi\left(t_{2}\right)-\varphi\left(t_{1}\right)\right| \leqq L\left|t_{2}-t_{1}\right|^{\sigma},\left|\psi\left(t_{2}\right)-\psi\left(t_{1}\right)\right| \leqq L\left|t_{2}-t_{1}\right|^{\sigma}$, $t_{1}, t_{2} \in\langle 0, b\rangle$, then the solution $u=u(x, t ; \varphi, 0, \psi)$ of the equation (9) satisfies 
the inequality

$$
\mid u\left(x_{2}, t_{2} \mid-u\left(x_{1}, t_{1}|| \leqq r(\sigma) L\left(\left|x_{2}-x_{1}^{12 \sigma}+\right| t_{2}-\left.t_{1}\right|^{\sigma}\right)\right.\right.
$$

for any two points $\left(x_{1}, t_{1}\right),\left(x_{2}, t_{2}\right) \in E$.

Lemma 4 ([11], p. 363). - Let $\left.\chi \in C_{0} \mid<0, a>\right), \chi_{(0)}=\chi(a)=0$. Let, further, there exist numbers $0<\alpha \leqq 1, M>0$ such that $\left|\chi\left(x_{2}\right)-\chi\left(x_{1}\right)\right| \leqq M \mid x_{2}-$ $\left.x_{1}\right|^{x}$ for any two points $x_{1}, x_{2} \in\langle 0, a\rangle$.

Then the solution $u=u(x, t ; 0, \chi, 0)$ of the differential equation (9) possesses the following properties:

a) $\left.\mid u_{2}^{\prime} x_{2}, t\right)-u\left(x_{1}, t\right)|\leqq M| x_{2}-\left.x_{1}\right|^{\alpha},\left(x_{1}, t\right),\left(x_{2}, t\right) \in E$,

b) $\left|u\left(x, t_{2}\right)-u\left(x, t_{1}\right)\right| \leqq \frac{2^{2+x} M}{\sqrt{\pi}} \int_{0}^{\infty} w^{x} e^{-w^{2}} d w\left|t_{2}-t_{1}\right|^{\alpha / 2}$

for any two points $\left(x, t_{1}\right),\left(x, t_{2}\right) \in E$.

Proof of Theorem 4. In the Banach space $C_{0}(E)$ where the norm is, as usually, defined by $\|v\|=\max |v(x, t)|$ we define (similarly as in [9]) the operator $T$ in the following way. For each $v \in C_{0}(E)$ we consider the function $r$ given by

$$
\begin{gathered}
r(x, t)=\frac{1}{2 \sqrt{\pi}} \int_{0}^{t} \int_{0}^{a} \frac{\left.\exp \left[-(x-\xi)^{2} / 4 \mid t-\tau\right)\right]}{\sqrt{t-\tau}} f[\xi, \tau, v(\xi, \tau)] d \xi d \tau \\
0 \leqq x \leqq a, 0<t \leqq b \\
r(x, 0)=0,0 \leqq x \leqq a
\end{gathered}
$$

and the solution $q$ of the equation (9) $q=u(x, t ; \varphi-r(0, t), \chi, \psi-r(a, t))$. Then we put

$$
T(v)=r+q=w
$$

We also denote the equality (15) by $w=T(v ; f, \varphi, \chi, \psi)$, where $f, \varphi, \chi, \psi$ play the role of parameters. From the definition of $w$ it follows that $w \in C_{0}(E)$ and $w_{\mathrm{i},}$ satisfies the conditions (12).

To prove that $w$ satisfies a Hölder condition in $x$ of the exponent $\alpha$ uniformly with respect to $t$ in $E$, let us consider the decomposition (15). The function $r$ shows this property on basis of Lemma 2 and the remark before Theorem 1. When we introduce the functions $\chi_{1}(x)=\chi_{(0)}+[\chi(a)-\chi(0)] \frac{x}{a}, 0 \leqq$ 
$x \leqq a, \varphi_{1}(t)=\varphi(t)-r(0, t)-\varphi(0), \psi_{1}(t)=\psi(t)-r(a, t)-\psi(0), 0 \leqq t \leqq b$, we can write

$$
q(x, t)=\chi_{1}(x)+u\left(x, t ; 0, \chi-\chi_{1}, 0\right)+u\left(x, t ; \varphi_{1}, 0, \psi_{1}\right) .
$$

From the assumption $\chi \in H_{a}(<0, a>)$, in virtue of Lemma 4, it follows that $u\left(x, t ; 0, \chi-\chi_{1}, 0\right)$ satisfies a Hölder condition in $x$ of the exponent $\alpha$ aniformly with respect to $t$ in $E$. Lemma 2 gives that the functions $\xi_{1}, \psi_{1} \in$ $H_{\sigma}(<0, b>)$, where $\sigma \leqq \alpha$, therefore it may be assumed that $0<\frac{\alpha}{2} \leqq \sigma<\frac{1}{2}$.

Then, by Lemma $3, u\left(x, t ; \varphi_{1}, 0, \psi_{1}\right)$ satisfies a Holder condition in $x$ of the exponent $2 \sigma$ and hence of the exponent $\alpha$, too, uniformly with respect to $t$ in $E$. Similarly we get that $w$ satisfies a Hölder condition in $t$ of the exponent $\frac{\alpha}{2}$ uniformly with respect to $x$ in $E$. Therefore there exists a constant $M>0, M=M(v)$ such that $w$ satisfies the inequality (13) for any two points $\left(x_{1}, t_{1}\right),\left(x_{2}, t_{2}\right) \in E$. From that property of $w$ it follows that if $T(z)=z$, then the function $f[x, t, z(x, t)]$ is locally Holder cotinuous in $x$ uniformly with respect to $t$ in $E$ and hence, on the basis of Theorem 9, [3], p. 21, $z$ is a solution of the problem (11), (12). Therefore it suffices to prove the existence of a fixed point of the operator $T$. To that aim let us consider some properties of the operator $T$.

By the continuity of $f$ in $D$ the function $r$ continuously depends on $v$. With respect to the continous dependence of the solution $q$ of the equation (9) on the initial and boudary conditions this implies that the operator $T$ continuously maps $\left.C_{0} ! E\right)$ into itself. When a sequence $v_{n} \in C_{0}(E)$ is bounded, then again by the continuity of $f$ there exists a constant $M>0$ such that $\left|f\left(x, t, v_{n}(x, t)\right)\right| \leqq M$ for each $(x, t) \in E$ and on basis of Lemma 2 the corresponding sequence of $\boldsymbol{r}_{n}$ given by (14) where instead of $v$ we put $v_{n}$ is uniformly bounded and equicontinnous. Hence, there exists a subsequence $r_{n_{k}}, k=1,2$, $3, \ldots$ which is uniformly convergent on $E$ to a function $r \in C_{0}(E)$. Let $q_{n}$ be the solution of the differential equation $(9), q_{n}=u\left(x, t ; \varphi-r_{n}(0, t), \chi, \psi-r_{n}(a, t)\right)$. From the continuous dependence of the solutions of (9) on the initial and the boundary conditions we get that $q_{n_{k}}$ uniformly converge on $E$ to the solution $q$ of the equation (9) given by the relation $q=u(x, t ; \varphi-r(0, t), \chi, \psi-r(a, t))$. Thus the sequence $T\left(v_{n_{k}} ; \varphi, \chi, \psi\right)$ is convergent in $C_{0}(E)$ to $q+r$ and the operator $T$ is completely continuous. Its further property is that for every bounded set $S \subset C_{0}(E)$ there exists a constant $K=K(S)>0$ such that

$$
\|T(v ; p, \chi, \psi)\| \leqq K
$$

for each $v \in S$. This follows from the uniform boundedness of the functions 
(14) corresponding to the functions $v \in S$ and from the maximum (and minimum) principle for the solutions of the equation (9).

Let us, now, consider the system of the operators $T_{k}, 0 \leqq k \leqq 1$, defined in $C_{0}(E)$ by the rulation $T_{k}(v)=T(v ; k f, k \varphi, k \chi, k \psi)$. From the construction of the operator $T$ we see that $T_{k}(v)=k T(v)$ for each $v \in C_{0}(E)$ and $\left.k \in<0,1\right\rangle$. Then the following statements are true:

a) For each $k, 0 \leqq k \leqq 1, T_{k}$ is a continuous mapping of $C_{0}(E)$ into itself.

b) If $S \subset C_{0}(E)$ is an arbitrary bounded set and $v \in S$, then $\left\|T_{k_{2}}(v)-T_{h_{1}}(v)\right\|$ $=\left|k_{2}-k_{1}\right| \cdot\|T(v)\|$ what, on the basis of $(16)$, implies the uniform continuity of $T_{k}$ in $k$ on $S$.

c) For any fixed $k, 0 \leqq k \leqq 1, T_{k}$ is a completely continuous operator.

d) If $v=T_{k}(v)$, then $v$ satisfies the conditions

$$
\begin{gathered}
v(0, t)=k \varphi(t), v(a, t)=k \psi(t), 0 \leqq t \leqq b \\
v(x, 0)=k \chi(x), 0 \leqq x \leqq a
\end{gathered}
$$

and similarly as in the case of the solution of the functional equation $z=T(z)$ it can be shown that $v$ satisfies the differential equation

$$
\frac{\partial v}{\partial t}=\frac{\partial^{2} v}{\partial x^{2}}+k f(x, t, v)
$$

Since the function $k f$ also satisfies the inequality (10), by Lemma 1 there exists a constant $M_{1}>0$ independent on $k$ such that $\|v\| \leqq M_{1}$.

e) The equation $v=T_{0}(v)$ has only the trivial solution.

We see that all assumptions of the Leriy-Sehauder theorem ([3], p. 189) are satisfied and, by that theorem, the operator $T$ has at least one fixed point in $C_{0}(E)$.

4. - Finally we proceed to the main theorem of that paper. The proof of that theorem will be based on the following lemmas.

Lemma 5. - Let the function $p=p(t, y)$ satisfy the condition $(A)$, the function $f=f(x, t, y)$ be continuous in $D$ and satisfy the inequality (10). Let further the functions $\varphi, \psi \in C_{0}(<0, b>), \chi \in C_{0}(<0, a>), \chi_{(0)}=\varphi(0), \chi(a)=\psi(0)$. Let the number $\alpha$ satisfy the inequalities $0<\alpha<1$. Then there exist:

1. A sequence of functions $\left\{f_{n}\right\}_{n=1}^{\infty}, f_{n}=f_{n}(x, t, y) \in H_{a}\left(D_{m}\right)$ for each $m=1,2,3, \ldots$, which is aniformly convergent to $f$ on each $D_{m}, m=1,2,3, \ldots$;

2. Sequences $f_{n}, \psi_{n} \in H_{a}(<0, b>), \chi_{n} \in H_{a}(<0, a>)$ such that $\chi_{n}(0)=$ 
$\varphi_{n}(0), \chi_{n}(a)=\psi_{n}(0)$ which uniformly converge on the corresponding intervals to the functions $\varphi, \psi$ and $\chi$, respectively;

3. A sequence of the solutions $z_{n}$ of the first initial-boundary value problem

$$
\begin{gathered}
\frac{\partial z}{\partial t}=\frac{\partial z}{\partial x^{2}}+f_{n}(x, t, z), 0<x<a, 0<t<b \\
z(0, t)=\varphi_{n}(t), z(a, t)=\psi_{n}(t), 0 \leqq t \leqq b, z(x, 0)=\chi_{n}(x), 0 \leqq x \leqq a
\end{gathered}
$$

which satisfy the inequality (13) with constants $M=M(n)$ for any two points $\left(x_{1}, t_{1}\right),\left(x_{2}, t_{2}\right) \in E$. This sequence is uniformly convergent on $E$ to a function $z \in C_{0}(E)$ which satisfies the conditions (12) and pussesses the continuous $\frac{\partial z}{\partial x}$ in $E_{0}$.

Proof. - By Theorem 3 there exists $q$ which satisfies the condition $(A)$ whereby $q \in H_{1}\left(B_{0, m}\right)$ for each $m=1,2,3, \ldots$ and $q(t, y)>p(t, y)$ in $B$. On the basis of Theorem 2 we get the existence of sequences of functions $f_{n}, \varphi_{n}, \psi_{n}$, and $\chi_{n}$ with the properties mentioned in the statement of the last theorem. $f_{n}$ satisfy the inequality

$$
\left|f_{n}(x, t, y)\right| \leqq q(t, \mid y),(x, t, y) \in D, n=1,2,3, \ldots
$$

The existence of a solution $z_{n}$ of the boundary value problem (17), (18) as well as the validity of the inequality (13) for this solution follows from Theorem 4 by using the inequalities (19). From the proof of that theorem we can see that $z_{n}$ can be got as the solution of the functional equation

$$
z_{n}=r_{n}+q_{n}
$$

where $q_{n}$ is determined by the equality (14) in which instead of $f$ and $v$ we put $f_{n}$ and $z_{n}$, respectively, and $g_{n}(x, t)=u\left(x, t ; \varphi_{n}-r_{n}(0, t), \chi_{n}, \psi_{n}-r_{n}(a, t)\right)$. Since the sequences of functions $\varphi_{n}, \psi_{n}$ and $\chi_{n}$ are uniformly bounded, in view of Lemma 1 the same property is shared by the sequence of $z_{n}$ on $E$. Lemma 2 then gives that the sequence of functions $r_{n}$ is uniformly bounded and equicontinuous. Hence there exists a subsequence $r_{n_{k}}$ which is uniformly convergent on $E$. With that sequence $q_{n_{k}}$ is also uniformly convergent. If we now consider only the subsequences $f_{n_{k}}, \varphi_{n_{k}}, \psi_{n_{k}}, \chi_{n_{k}}, z_{n_{k}}$ and denote them by $f_{n}, \varphi_{n}, \psi_{n}, \chi_{n}$, and $z_{n}$, respectively, we obtain the statement of the theorem. The continuity of $\frac{\partial z}{\partial x}$ follows from the decomposition $z=r+q$ where $q$ is a suitable solution of the equation (9) and $r$ is determined by the equality (14) 
whereby instead of $v$ acts $z$. Since the composite function $f[x, t, z(x, t)] \in C_{0}(E)$, $\frac{\partial r}{\partial x}$ is continuous in the interior of $E([3]$, p. 21).

Further properties of the sequences $\left\{z_{n}\right\}$ mentioned in Lemma 5 will be derived from the following lemma based on Theorem 7, [7], p. 33 .

LEMMa 6. - If the function $u=u(x, t)$ together with the derivatives $\frac{\partial u}{\partial x}$, $\frac{\partial^{2} u}{\partial x^{2}}, \frac{\partial u}{\partial t}$ is continuous on $E$ and in $E_{0}$ satisfies the equation

$$
\frac{\partial u}{\partial t}=\frac{\partial^{2} u}{\partial x^{2}}+h(x, t) \quad\left(h \in C_{0}(E)\right)
$$

as well as the inital-boundary conditions

$$
u(x, 0)=0,0 \leqq x \leqq a, u(0, t)=u(a, t)=0 ; 0 \leqq t \leqq b,
$$

then there exists a constant $K>0$, depending only on $a, b$ such that

$$
\iint\left|u_{E}^{2}(x, t)+\left(\frac{\partial u(x, t)}{\partial t}\right)^{2}+\left(\frac{\partial u(x, t)}{\partial x}\right)^{2}+\left(\frac{\partial^{2} u(x, t)}{\partial x^{2}}\right)^{2}\right| d x d t \leqq K \iint_{E} h^{2}(x, t) d x d t
$$

Proor. - By the mentioned Theorem 7, there exist two constants $M_{1}>0$, $M_{2}>0$ depending only on $a, b$, such that for every $\tau, 0 \leqq \tau \leqq b$,

$$
\begin{aligned}
& \int_{0}^{a} u^{2}(x, \tau) d x+\int_{0}^{\tau} \int_{0}^{a}\left[\frac{\partial u(x, t)}{\partial x}\right]^{2} d x d t \leqq M_{1} \int_{0}^{\tau} \int_{0}^{a} h^{2}(x, t) d x d t \\
& \int_{0}^{a}\left[\frac{\partial u(x, \tau)}{\partial x}\right]^{2} d x+\int_{0}^{\tau} \int_{0}^{a}\left[\frac{\partial u(x, t)}{\partial t}\right]^{2} d x d t \leqq M_{2} \int_{0}^{\tau} \int_{0}^{a} h^{2}(x, t) d x d t .
\end{aligned}
$$

From these two inequalities, in view of the inequality

$$
\int_{0}^{\tau}\left[\int_{0}^{u} \int_{0}^{a} h^{2}(x, t) d x d t\right] d u=\int_{0}^{\tau} \int_{0}^{a}(\tau-t) h^{2}(x, t) d x d t \leqq b \int_{0}^{\tau} \int_{0}^{a} h^{2}(x, t) d x d t
$$

and the equation (20), the inequality (22) follows.

Finally, we come to the main theorem.

Theorem 5. - Let the function $p=p(t, y)$ satisfy the condition $(A)$, let the function $f=f(x, t, y)$ be continuous in $D$ and satisfy the inequality (10). 
Let further the functions $\varphi, \psi \in C_{0}(<0, b>), \chi \in C_{0}(<0, a>)$, and $\chi(0)=\varphi(0)$, $\chi(a)=\psi(0)$. Denote $f(0,0, \varphi(0))=c_{1}, f(a, 0, \psi(0))=c_{2}$. Then for every $\delta, 0<\delta$ $<\frac{1}{2}$, there exists a function $z \in C_{0}(E)$ with $\frac{\partial z}{\partial x} \in C_{0}\left(E_{0}\right)$ and having the weak derivatives $\frac{\partial^{2} z}{\partial x^{2}}, \frac{\partial z}{\partial t}$ such that:

1. $z=z_{1}+z_{2}$,

where $z_{1} \in \bar{C}_{1+\delta}(E) \cap W^{2,1}(E)$ and $z_{1}$ satisfies almost everywhere (a.e.) in $E_{0}$ the differential equation

$$
\frac{\partial z_{1}}{\partial t}=\frac{\partial^{2} z_{1}}{\partial x^{2}}+f\left(x, t, z\left(x, t_{i}\right)-\left(c_{1}+\frac{c_{2}-c_{1}}{a} x\right)\right.
$$

and everywhere the conditions $(21) ; z_{2} \in C_{2+1}\left(E_{0}\right)$ is the (classical) solution of the equation

$$
\frac{\partial z_{2}}{\partial t}=\frac{\partial^{2} z_{2}}{\partial x^{2}}+c_{1}+\frac{c_{2}-c_{1}}{a} x
$$

which satisfies the conditions (12). where.

2. $z$ satisfies the equation (11) in $E_{0}$ a.e and the conditions (12) every.

Proof. - For the given $\delta, 0<\delta<\frac{1}{2}$, put $\alpha=\sqrt{2 \bar{\delta}}$. Then $0<\alpha<1$, and by Lemma 5 , there exists a sequence of solutions $z_{n}$ of the problem (17), (18) which is uniformly convergent on $E$ to a function $z \in C_{0}(E)$ with $\frac{\partial z}{\partial x}$ $\epsilon C_{0}\left(E_{0}\right)$. We shall prove that $z$ has all properties mentioned in this theorem.

Preserving the notations from Lemma 5, for every $n=1,2,3, \ldots$ and every $(x, t) \in E$ we denote

$$
\begin{gathered}
g_{n}(x, t)=f_{n}\left(x, t, z_{n}(x, t)\right), h_{n}(x, t)=g_{n}(x, t)-\left[g_{n}(0,0)+\frac{g_{n}(a, 0)-g_{n}(0,0)}{a} x\right] \\
g(x, t)=f(x, t, z(x, t)), h(x, t)=g(x, t)-\left(c_{1}+\frac{c_{2}-c_{1}}{a} x\right)
\end{gathered}
$$

Thus, $h_{n}(0,0)=h_{n}(\alpha, 0)=0$ and, by Lemma 5 , the sequences $\left\{g_{n}\right\}_{n=1}^{\infty}$, $\left\{h_{n}\right\}_{n=1}^{\infty}$ uniformly converge on $E$ to $g$ and $h$, respectively. Also each $g_{n}$ as well as $h_{n}$ belongs to $H_{\delta}(E)$.

Let $z_{1, n}$ be the solution of the differential equation

$$
\frac{\partial z}{\partial t}=\frac{\partial^{2} z}{\partial x^{2}}+h_{n}(x, t), 0<x<a, 0<t<b,
$$


which satisfies (21). By Theorem 6, [3], p. 124, $z_{1, n}$ exists and is unique. Theorem 4, [3], p. 191, assures the existence of a constant $K>0$ depending only on $\delta, a, b$ such that $\overline{\left.\left|z_{1, n}\right|\right|_{1} ^{E_{0}}+\delta} \leqq K, h_{n} !_{0}^{E_{0}} \leqq K_{1}$. Since each Hölder continuous function on $E_{0}$ can be extended to $E$ as Hölder continuous with the same Hölder coefficient and the derivative of such a function at a boundary point $p$ of $E_{0}$ is equal to the limit at $p$ of that derivative, we also have $\left|z_{1, n}\right|{ }_{1+\delta}^{E} \leqq K_{1}$. By this inequality both sequences $\left\{z_{1, n}\right\},\left\{\frac{\partial z_{1, n}}{\partial x}\right\}$, are uniformly bounded and equicontinuous on $E$. Hence there exists a function $z_{1} \in \bar{O}_{1+\delta}(E)$ satisfying (21) and a subsequence $\left\{z_{1, n_{k}}\right\}_{k=1}^{\infty}$ which uniformly converges to $z_{1}$, and $\left\{\frac{\partial z_{1, n_{k}}}{\partial x}\right\}$ uniformly converges to $\frac{\partial z_{1}}{\partial x}$, both on E. Similarly, as in Lemma 5 , we denote this sequence again by $\left\{z_{1, n}\right\}_{n=1}^{\infty}$. The same change of notation will be referred to all other sequences which we are dealing with. From Theorem 7 , [3], p. 65, it follows that each $z_{1, n} \in \bar{C}_{2+\delta}(E)$ and thus we can apply Lemma 6 to $z_{1, n}$. By this lemma, there is a constant $K_{2}>0$ such that

$$
\iint\left[z_{1, n}^{2}(x, t)+\left(\frac{\partial z_{1, n}(x, t)}{\partial t}\right)^{2}+\left(\frac{\partial z_{1, n}(x, t)}{\partial x}\right)^{2}+\left(\frac{\left.\partial^{2} z_{1, n} \mid x, t\right)}{\partial x^{2}}\right)^{2}\right] d x d t \leqq K_{2}
$$

for all $n=1,2,3, \ldots$. From that, on the basis of Lemma 6.2, [4], p. 16, wo get that $z_{1} \in W^{2,1}(E)$ and $\left\{\frac{\partial z_{1, n}}{\partial t}\right\},\left\{\frac{\partial^{2} z_{1, n}}{\partial x^{2}}\right\}$ converge weakly (in $L^{2}(E)$ to the weak derivatives $\frac{\partial z_{1}}{\partial t}$ and $\frac{\partial^{2} z_{1}}{\partial x^{2}}$, respectively. Hence, by going to the limit in (25), as $n \rightarrow+\infty$, we obtain that $z_{1}$ satisfies (23) in $E_{0}$ a .e. It also fulfils the conditions (21).

Let $z_{2, n}$ be the function given by

$$
\begin{gathered}
z_{2, n}(x, t)=\left(g_{n}(0,0)+\frac{g_{n}(a, 0)-g_{n}(0,0)}{a} x\right) t+u\left(x, t ; \varphi_{n}(t)-g_{n}(0,0) t\right. \\
\left.\chi_{n}(x), \psi_{n}(t)-g_{n}(a, 0) t\right)
\end{gathered}
$$

that is, the unique solution of

$$
\frac{\partial z}{\partial t}=\frac{\partial^{2} z}{\partial x^{2}}+g_{n}(x, t)-h_{n}(x, t), 0<x<a, 0<t<b,
$$

which satisfies the conditions (18). Corollary to Theorem 15, [3], p. 80, gives that $\left\{z_{2, n}\right\}$ is uniformly convergent on $E$ to the funotion $z_{2}$, given by

$$
z_{2}(x, t)=\left(c_{1}+\frac{c_{2}-c_{1}}{a} x\right) t+u\left(x, t ; \varphi(t)-c_{1} t, \chi(x), \psi(t)-c_{2} t\right)
$$


and hence $z_{2}$ is the unique solution of the equation (24) which satisfies the conditions (12). By Theorem 9, [3], p. 69, $z_{2} \in C_{2+1}\left(E_{0}\right)$.

Since $z_{1, n}+z_{2, n}$ satisfy the conditions (18) and the equation $\frac{\partial z}{\partial t}=\frac{\partial^{2} z}{\partial x^{2}}+$ $g_{n}(x, t)$, thus, the same conditions as $z_{n}$, we have $z_{1, n}+z_{2, n}=z_{n}$, and thus, $z_{1}+z_{2}=z$. Patting the result for $z_{1}, z_{2}$ together, we finally see that $z$ satisfies the equation (11) in $E_{0}$ a.e. and the conditions (12).

\section{REFERENCES}

[1] C. Crciberto, Su di un problema al contorno per una equazione non lineare di tipo parabolico in due variabili, Ricerche Mat. 1 (1952), pp. 50̃-77.

[2] - -, Su di un problemz al contorno per l'equazione $u_{x x}-u_{y}=f\left(x, y, u, u_{x}\right)$, Ricerche Mat. 1 (1952), pp. $295-316$.

[3] A. Erredman, Partial differential equations of parabolic type, Prentice Hall 1964.

[4] - -, Partial lifferential equations, Holt, Rinehart and Winston, Ine. 1969.

[5] E. GAGLARDO, Problema al contomo per equazioni differenziali lineari di tipo para. bolico in $n$ variabili, Ricerche Mat. 5 (1956), pp. 169-205.

[6] - - Teoremi di esistenza e di unicità per problemi al contorno relativi ad equazio. ni paraboliche lineari e quasi lineari in $n$ variabili, Ricerche Mat. 5 (1956) pp. 239-257.

[7] A. M. ILJIN, A. S. Kalasinikov, O. A. OLEJNIK, Linear equations of the second order of parabolic type (In Russian), Usp. Mat. Nauk 17 (1962), pp. 3-146.

[8] E. KAMke, Differentialgleichungen reeler Funktionen, Leipzig 1956.

[9] W. MLAK, The first boundary value problem for a nonlinear parabolic equation, Ann. Pol. Math. 5(1958), pp. 257-272.

[10] O. A. OLEJNIK, S. N. KRUZKov, Quasi-linear parabolic equations of the second order with many independent variables (In Russian), Usp. Mat. Nauk, 16(1961), pp. 115-155.

[11] V. Seda, A remark to the theory of the heat equation, Ann. Mat. Pura Appl. 86 (1970), pp. 357.366 . 